behavioural, and biological factors. C. trachomatis genotype L2 was detected in women with PID.

\section{P3.37 THE ROLE OF ENGAGEMENT WITH PARTNER NOTIFICATION IN UNDERSTANDING STI DIAGNOSIS INEQUALITIES ACROSS ETHNIC GROUPS: EVIDENCE FROM A LARGE PATIENT SURVEY IN ENGLAND}

Cath Mercer, ${ }^{1,2}$ S Wayal, ${ }^{1,2}$ S Fabiane, P Blomquist ${ }^{1,3}$, D Reid ${ }^{1,4}$, P Weatherburn ${ }^{1,4}$ G Hughes ${ }^{1,4}$. 'National Institute of Health Research (NIHR) Health Protection Research Unit (HPRU) in Blood Borne and Sexually Transmitted Infections, United Kigdom; ${ }^{2}$ Centre for Sexual Health and HIV, Research Department of Infection and Population Health, University College London, United Kigdom; 3 London School of Hygiene and Tropical Medicine, London, United Kigdom; ${ }^{4}$ Public Health England, United Kigdom

\subsection{6/sextrans-2017-053264.274}

Introduction STI diagnosis rates vary considerably by ethnicity in England and persist after adjusting for confounding factors including deprivation and sexual behaviour. We examine the extent to which partner notification (PN) experiences differ by ethnicity as a possible contributing factor to this health inequality.

Method 3986 patients attending 17ethnically-diverse sexual health clinics in England, between May and September 2016, self-completed an online survey, which included questions onsociodemographics, sexual behaviour, and PN experience. Prior to survey analyses, these data were linked to clinic data on STI diagnosis/es and services received at their clinic visit. Age-adjusted ORs (AORs) were calculated for the 6main ethnic minority groups in England(Black Caribbean, Black African, Asian, Mixed, and White other, and other) relative to White British patients (36\% of the sample).

Results Overall, 25\% of men and 20\% of women reported STI diagnosis/es(past year), but this was higher among those of mixed ethnicity, Black Caribbeans and 'White others', (AORs:1.47, 1.40,1.27respectively). Of patients reporting STI diagnoses, $75 \%$ said that clinic staff advised them to inform their partners to test for STIs, while $60 \%$ of patientsdid actuallynotify all their partners. Reporting of both of these PN measures was higher among Black Caribbeans(AORs:2.05 and 1.92, respectively) and those of mixed ethnicity (AORs:1.92 and 1.59, respectively). Of those who had not informed all their partners, 69\% of women and 55\% of men reported condomless last sex, with this significantly higher for women (only) of Black Caribbean or mixed ethnicity (AOR:2.52 and 5.81, respectively). Partner numbers were larger for those who had not informed all partners: $40 \%$ reported $5+$ (past year) vs. $31 \%$ of those who had; this did not vary significantly by ethnicity.The 3 mostcommonly reportedreasons for not informing partners were: not having their contact details (66\%), embarrassment (57\%), and not being concerned about notifying casual/one-off partners (54\%).

Conclusion Overall, engagement with $\mathrm{PN}$ is relatively high and inequalities in PN experience do not appearto explain disproportionate STI risk in some ethnic groups. However, among those who did not notify all their partners STI risk behaviour was more commonly reported,suggesting efforts toimprove PN should be maintained. The development of strategies for empowering black Caribbean and mixed-ethnicity women to negotiate safer sex and condom use is a priority.

\section{P3.38 USE OF RAPID DIAGNOSTICS FOR CHLAMYDIA AND GONORRHOEA FOR WOMEN IN THE EMERGENCY DEPARTMENT CAN IMPROVE CLINICAL MANAGEMENT: REPORT OF A RANDOMISED CLINICAL TRIAL}

Charlotte Gaydos, Mitra Lewis, Michele-Corinne Ako, Richard Rothman, Andrea Dugas. Johns Hopkins University, Baltimore, USA

\subsection{6/sextrans-2017-053264.275}

Introduction In the Emergency Department (ED), accurate diagnosis and appropriate treatment of Chlamydia trachomatis (CT) and Neisseria gonorrhoea (NG) is a challenge. Lengthy routine result times for $\mathrm{CT} / \mathrm{NG}$ tests often lead to empiric antibiotic over treatment or lack of treatment of infections. A randomised clinical trial was conducted to determine how use of rapid diagnostics could improve clinical management. The objective was to assess the effects of rapid CT/NG testing on over treatment and under treatment of women being evaluated for sexually transmitted infections (STIs) in the ED.

Methods Women undergoing pelvic examinations and STI testing in the $\operatorname{ED~}(n=253)$ were consented and randomised to either a control or rapid testing group. The control group received standard of care (SOC), with CT/NG testing by nucleic acid amplification tests (NAAT) with a 2-3 day turnaround time. Patients in the rapid testing group provided an additional vaginal swab used for rapid Gene Xpert CT/NG testing with a $100 \mathrm{~min}$ turnaround time. Results from the rapid tests were presented to providers and patients were treated according to clinical judgment. Following discharge there was a 2 week phone follow-up and chart review.

Results $100 \%$ of CT positive $(n=9)$ and $100 \%$ NG positive $(\mathrm{n}=5)$ patients in the rapid testing group received appropriate antibiotic treatment as compared to $54 \%$ (7/13) CT positive patients $(p=0.046)$ and $43 \% \quad(3 / 7) \quad N G$ positive patients $(\mathrm{p}=0.081)$ in the control group. Additionally, in the control group, $36.8 \%$ (42/114) of CT negative and 38.3\%, (46/120) of NG negative patients were over treated.

Conclusions Both under- and over treatment for CT/NG was observed in the SOC control group. Patients with positive results in the rapid test group were more appropriately treated. This study demonstrated the potential clinical impact and subsequent antibiotic stewardship of using rapid CT/NG testing the ED.

\section{P3.39 MOLECULAR DETECTION OF TRICHOMONAS VAGINALIS VIRUS IN DIRECT TRICHOMONAS VAGINALIS POSITIVE CLINICAL SAMPLES FROM THE NETHERLANDS}

${ }^{1} \mathrm{C}$ Van der Veer, ${ }^{1} \mathrm{I}$ Jehee, ${ }^{1} \mathrm{M}$ Himschoot, ${ }^{2} \mathrm{M}$ Hermans, ${ }^{1} \mathrm{~S}$ Bruisten. ${ }^{1}$ Public Health laboratory, GGD Amsterdam, the Netherlands; 2 Jeroen Bosch Ziekenhuis (JBZ) in 'S Hertogenbosch, the Netherlands

\subsection{6/sextrans-2017-053264.276}

Introduction Two genotypes are described for Trichomonas vaginalis (TV). TV genotype I seems to be more susceptible to metronidazole, but also more prone to TV virus (TVV) infection, than type II. The release of TVV during treatment may in itself be pathogenic. Four TVV genotypes have been described, but epidemiological studies are rare as culturing TV ahead of TVV detection is laborious. We therefore developed a sensitive method to detect and type TVV in TV positive clinical samples directly. 
Methods TV positive samples (by NAAT) were collected from two Public Health Laboratories in the Netherlands, from 2012 to 2016. TV was typed using multi-locus sequence typing (MLST) of 7 household genes. MLST profiles were put into a minimum spanning tree together with reference strains and so allocated a genotype. TVV RNA was detected using nested reverse-transcriptase PCR with newly designed primers and visualised on gel.

Results We included 157 clinical samples from 151 clients (most were female; $n=133,87 \%$ ). In total, 120 samples were genotyped for TV; TV genotype I was most common $(n=65 ; 54 \%)$. Over half of these samples were found positive for TVV RNA $(n=65 ; 54 \%)$. Most TVV infections were found in TV genotype I $(\mathrm{n}=49 ; 75 \%)$. The amplified products for TVV1, TVV2 and TVV3 were respectively $97 \mathrm{bp}, 290 \mathrm{bp}$ and $156 \mathrm{bp}$ and sequencing showed high identity with TVV genomes; TVV1-C344 (93\%), TVV2-OC3 (89\%) and TVV3UR1 $(85 \%)$, supporting specificity of the primers for these targets. TVV1 was most prevalent $(n=45 ; 41 \%)$, followed by TVV3 $(n=34 ; 31 \%)$ and finally TVV2 $(n=31 ; 28 \%)$. Co-infections occurred in $60 \%$ of TVV positive samples. TVV4 was not found at all. Sensitivity for TVV detection still needs to be established using TV samples with a known TVV (genotype) status.

Conclusion TVV detection can be performed directly on clinical samples using nested RT-PCR. TVV infection occurs most frequently in TV type I parasites as in agreement with previous findings. This opens the way for molecular epidemiological studies to gain insight into the role of TVV in TV pathogenicity.

\section{P3.40 SOCIO DEMOGRAPHIC AND SEXUAL BEHAVIOURAL CORRELATES OF HERPES SIMPLEX VIRUS TYPE 2 INFECTION AMONG PREGNANT WOMEN IN SOUTHWESTERN NIGERIA}

${ }^{1}$ Chinenye Gloria Anaedobe, ${ }^{2}$ Fowotade Adeola, ${ }^{3}$ Bakare Rasheed Ajani. 1. University of Abuja, Federal Capital Territory, Abuja, Nigeria; ${ }^{2}$ University College Hospital, Ibadan, Ibadan, Nigeria; ${ }^{3}$ University of Ibadan, Ibadan, Nigeria

\subsection{6/sextrans-2017-053264.277}

Introduction Herpes Simplex Virus type 2 (HSV 2) is the most common cause of genital ulcer disease and facilitates the acquisition of Human Immunodeficiency Virus (HIV). It leads to lifelong latent infection and this raises concerns among women of reproductive age, considering the risk of neonatal transmission. This study aims to determine HSV-2 seroprevalence among pregnant women, identify the correlation with socio-demographic characteristics, sexual behaviour, pregnancy outcomes and co-infection with HIV.

Methods A total of 270 pregnant women attending antenatal clinic of the University College Hospital Ibadan were enrolled in this cross-sectional study. Their serum samples were tested for HSV-2 IgG using type specific third generation ELISA and HIV1, using Uni-Gold Recombigen and ALERE determine. Pretested questionnaire were used to obtain bio-data on sociodemographic characteristics, sexual behaviour and obstetrics history of the participants. Data analyses was done using SPSS version 20 .

Results The seroprevalence of HSV 2 type specific IgG was $33.3 \%(90 / 270)$ and a HIV/HSV 2 co-infection rate of $39 \%$ (35/90) was observed. Logistic regression analysis showed that polygamy, low educational level, positive HIV status, previous sexually transmitted infections (STIs), early age at sexual debut and multiple sexual partners were independent risk factors for HSV-2 infection. Obstetrics complications such as intrauterine foetal death, congenital malformations and spontaneous abortion were predictors of HSV-2 infection.

Conclusion The seroprevalence of HSV-2 in this pregnant population is lower than what is observed in some other SubSaharan African countries; however, co-infection with HIV is high and majority of the women are still susceptible to primary HSV-2 infection in pregnancy. The demographic, sexual behaviour and bad obstetrics histories found to be predictors of HSV 2 infection in this study may be important in selecting candidates for screening tests, developing strategies towards effective health promotion campaign and reducing risk of HIV transmission.

\section{P3.41 PREVALENCE, SOCIO-DEMOGRAPHIC AND SEXUAL BEHAVIOURAL RISK FACTORS FOR HEPATITIS B VIRUS INFECTION AMONG PREGNANT WOMEN IN SOUTHWESTERN NIGERIA}

${ }^{1}$ Chinenye Gloria Anaedobe, ${ }^{2}$ Fowotade Adeola, ${ }^{3}$ Chukwuma E Omoruyi, ${ }^{4}$ Rasheed Ajani Bakare. 'University of Abuja, Federal Capital Territory, Abuja, Nigeria; ' University College Hospital, Ibadan, Ibadan, Nigeria; '3niversity College Hospital Ibadan, Ibadan, Nigeria; ${ }^{4}$ University of Ibadan, Ibadan, Nigeria

\subsection{6/sextrans-2017-053264.278}

Introduction In endemic countries, Hepatitis B virus (HBV) infection occurs mainly during infancy and early childhood, with vertical transmission from an infected mother being a major route of infection. This study aimed at identifying the prevalence and sexual behavioural risk factors for HBV infection among pregnant women in Ibadan, Nigeria.

Methods A cross-sectional study was done at the antenatal clinic of the University College Hospital Ibadan. One hundred and eighty pregnant women were recruited over a six month period, and their serum was tested for Hepatitis B surface antigen (HBsAg) (BIORAD FRANCE) using third generation ELISA and HIV-1 and 2, using Uni-Gold Recombigen and ALERE determine. Positive HBsAg samples were tested for Hepatitis B envelope antigen ( $\mathrm{HBeAg})$, antibody ( $\mathrm{HBeAb}$ ) and Hepatitis B core antibody (HBcAb) (DIAPRO Italy) while serum HBV DNA was detected using PCR. Data were obtained using questionnaires and analysed using SPSS-20

Results The seroprevalence of HBsAg was $8.3 \%$ out of which 26.7\% were positive for $\mathrm{HBeAg}, 53.3 \%$ had $\mathrm{HBeAb}, 20 \%$ had neither $\mathrm{HBeAg}$ nor $\mathrm{HBeAb}, 100 \%$ had total $\mathrm{HBcAb}$ and $86.7 \%$ had HBV DNA in their serum. A co-infection rate (HBV/HIV) of $26.7 \%$ was observed. The mean age was 32.1 years, the highest HBV infection rate occurred in 25-29 year age group. Multiple sexual partners $(\mathrm{OR}-3.987$, P- value $=0.026)$, early age at sexual debut $(\mathrm{OR}-11.996$, $\mathrm{P}$ - value $=0.022)$ were independent risk factors for HBV infection.

Conclusion The result of this study brings to light the high prevalence and high infectivity rate of chronic HBV virus infection among women of child bearing age in Nigeria. Thus, there is a dire need for routine screening for all pregnant women during antenatal care, immunoprophylaxis for exposed newborns and surveillance for those with chronic infection. The socio-demographic and risky sexual behavioural characteristics found to be predictors of HBV infection should be considered when developing strategies towards effective sexual 